\title{
Almacenes de biomasa y carbono aéreo y radicular en pastizales de Urochloa decumbens y Paspalum notatum (Poaceae) en el sureste de México
}

\author{
Eder Ramos-Hernández ${ }^{1}$ \& José Luis Martínez-Sánchez ${ }^{2 *}$
}

1. Instituto Nacional de investigaciones Forestales, Agrícolas y Pecuarias. Campo experimental Huimanguillo. Carretera Federal Humanguillo-Cárdenas Km. 1. 86400. Huimanguillo, Tabasco. México.

2. División Académica de Ciencias Biológicas, Universidad Juárez Autónoma de Tabasco, km 0.5 Carr. Vhs-Cárdenas, Villahermosa, Tab. 86660 México; jose.martinez@ujat.mx

*Correspondencia

Recibido 24-V-2019. Corregido 27-IX-2019. Aceptado 26-II-2020.

\begin{abstract}
Above and below-ground stocks of biomass and carbon in Urochloa decumbens and Paspalum notatum (Poaceae) pastures in southeastern Mexico. Introduction: Due to its current coverage, the root biomass of tropical pastures is of vital importance for the terrestrial carbon storage, but its estimation has been mainly indirect and with a very wide range of values. Objective: To document at national and global level as well as compare, the root carbon stocks extracted directly from two grasslands of different growth type and origin. Methods: The biomass and root carbon stocks were directly extracted from two tropical pastures with more than $40 \mathrm{yr}$ of age. The biomass and total carbon, one native with stoloniferous growth (Paspalum notatum) and one introduced with growth in tillers (Urochloa decumbens) were measured. The study was conducted between August and October 2016. Three plots of $1600 \mathrm{~m}^{2}$ each were selected per pasture, with 10 sub-squares $\left(4 \mathrm{~m}^{2}\right.$ each) in each plot to determine the floristic composition, aboveground biomass and $\mathrm{C}$. The biomass and root $\mathrm{C}$ were estimated by hand in three trenches $(1.50 \mathrm{~m} \times 0.50 \mathrm{~m} \times 1.0 \mathrm{~m})$ per plot. The organic carbon content was determined with the ignition method at an average temperature of $550{ }^{\circ} \mathrm{C}$ for 3 hours. For the statistical analysis, a two-factor ANOVA was used, where one factor was the treatments that were the type of pasture (2) and the other factor was the section of the grass (above and belowground). Results: The floristic composition of the two types of pasture was different due to its management history. On average, $28.25 \mathrm{Mg} \mathrm{ha}^{-1}$ of total C was estimated for the locality. The native pasture of stoloniferous growth $P$. notatum yields almost three-fold more biomass $\left(42.5 \mathrm{MgC} \mathrm{ha}^{-1}\right)$ than the introduced with growth in tillers $U$. decumbens $\left(14 \mathrm{MgC} \mathrm{ha}^{-1}\right)$ due to the radicular storage (38.5 vs. $11.46 \mathrm{MgC} \mathrm{ha}^{-1}$ ). Seventy-four point five percent of the carbon in P. notatum was located in the radicular part and $25.5 \%$ above-ground, while in $U$. decumbens 56.5 and $43.5 \%$, respectively. Conclusions: P. notatum accumulated more total and radicular biomass and carbon than $U$. decumbens. The best adaptation of the native grass as well as the morphology of its root system may explain this difference. Ninety six percent of the biomass and root $\mathrm{C}$ is found in the first $0.50 \mathrm{~m}$ depth. Due to the contribution of its radicular portion, tropical pastures can constitute important reservoirs of terrestrial carbon considering its extension nowadays.
\end{abstract}

Key words: Brachiaria decumbens, $\mathrm{C}_{4}$ pastures, soil profile, grassland, root biomass.

Ramos-Hernández, E., \& Martínez-Sánchez, J.L. (2020). Almacenes de biomasa y carbono aéreo y radicular en pastizales de Urochloa decumbens y Paspalum notatum (Poaceae) en el sureste de México. Revista de Biología Tropical, 68(2), 440-451. 
El establecimiento de pastizales (inducidos), principalmente para la ganadería, ha sido una de las principales causas del cambio de uso del suelo. Actualmente, la superficie de pastizales en México es de $196000 \mathrm{~km}^{2}$ (SEMARNAT, s.f.), y en el mundo de 39 millones de $\mathrm{km}^{2}$ (D'Atri, 2007) (35.8 millones de $\mathrm{km}^{2}$; Dixon, Faber-Langendoen, Josse, Morrison, \& Loucks, 2014).

Al igual que los bosques y selvas (Hughes, Kauffman, \& Jaramillo, 1999), los pastizales también pueden retener el carbono en la superficie terrestre y contribuir a la mitigación del efecto invernadero, aunque en menor magnitud (Houghton et al., 2000; Carrillo, Acosta, Flores, Juárez, \& Bonilla, 2014; Saraiva et al., 2014). Ni (2002). Por ejemplo, estimó satelitalmente en una región tropical en China un almacenamiento total de carbono en vegetación y suelo de pastizales de $3.88 \times 10^{9} \mathrm{Mg}$. Actualmente, debido al calentamiento global y a la retención del carbono en los ecosistemas terrestres, resulta de suma importancia estimar la productividad de biomasa y almacenamiento de carbono de los pastizales ganaderos tropicales. Con el fin de documentar y valorar la función ecológica de los pastizales en el mundo, se han realizado diversas estimaciones de su almacenamiento de carbono (Fan et al., 2008). La mayor parte de este tipo de datos provienen de la producción primaria neta, donde se ha documentado que los pastizales tropicales son más productivos (42-3 $396 \mathrm{~g} \mathrm{~m}^{-2}$ ) que los templados (45-532 $\mathrm{g} \mathrm{m}^{-2}$ ) (Gómez \& Gallopin, 1991). En pastizales alpinos de Paspalum spp. en Argentina, por ejemplo, se registran $2.64 \mathrm{Mg} \mathrm{ha}^{-1} \mathrm{de}$ biomasa aérea (Ruolo et al., no publicado), y $1.4 \mathrm{Mg} \mathrm{ha}^{-1}$ de biomasa de raíces a $1 \mathrm{~m}$ de profundidad en un pastizal de Cynodon nlemfuensis Vanderyst (Céspedes, Fernández, Gobbi, \& Bernardis, 2012). En Chihuahua e Hidalgo (México), respectivamente, las estimaciones en pastizales en zonas áridas y semiáridas documentan 4.9 y $2.0 \mathrm{Mg} \mathrm{ha}^{-1}$ de biomasa aérea (2.2 y 1.2 de C aéreo), 7.5 y $6.0 \mathrm{Mg} \mathrm{ha}^{-1}$ de biomasa radicular (2.5 y 3.5 de $C$ radicular), y 12.5 y 9 $\mathrm{Mg} \mathrm{ha}^{-1}$ de biomasa total $\left(5.0\right.$ y $6.0 \mathrm{Mg} \mathrm{ha}^{-1}$ de $\mathrm{C}$ total) (Yamallel et al., 2014; Montaño et al., 2016). En Coahuila, Hernández (2008) registran $5.26 \mathrm{Mg} \mathrm{ha}^{-1}$ y Castro (2008) $2.1 \mathrm{Mg}$ $\mathrm{C}$ ha-1. Para regiones tropicales existen menos datos disponibles. Giraldo, Zapata, \& Montoya (2006) reportan $38 \mathrm{Mg} \mathrm{C}^{-1}$ en pasto y suelo con Brachiaria dyctioneura (Fig. \& De Not.) Stapf, y Saraiva et al. (2014) $358 \mathrm{Mg} \mathrm{C} \mathrm{ha}^{-1}$ en pastizales de $B$. decumbens, ambos en Brasil.

Particularmente en las gramíneas, la biomasa subterránea o radicular suele ser igual o hasta $90 \%$ mayor que la biomasa superficial o aérea (Ávila, Jiménez, Beer, Gómez, \& Ibrahim 2001; Kanninen \& Mery, 2001), de tal manera que este componente adquiere gran importancia debido a su relevancia en el ciclo del carbono y la mitigación del $\mathrm{CO}_{2}$ atmosférico. Sin embargo, su estimación adecuada ha presentado cierto problema metodológico debido al esfuerzo, tiempo y costo de su extracción directa del suelo. Debido a esto, se ha estimado alométricamente (Barbaran, Rise, \& Alegre 2001), pero con un sesgo relativamente alto en los valores. En cuanto a los almacenes de biomasa radicular en los pastizales, Alegre, Arévalo, \& Ricse (2000) registraron 3.0, 1.92 y $1.36 \mathrm{Mg}$ $\mathrm{ha}^{-1}$ en Perú, y Jaramillo, Ahedo-Hernández, \& Kauffman (2003) encontraron de 3.1 a $5.45 \mathrm{Mg}$ $\mathrm{ha}^{-1}$ en Veracruz, ambos en pastizales de diferentes edades. Solamente López, Schlonvoight, Ibrahim, Kleinn, \& Kanninen (1999), Jaramillo et al. (2003) y de Koning, Velkamp, \& López Ulloa (2003) han detallado su distribución vertical hasta $1 \mathrm{~m}$ de profundidad.

El sureste de México se caracteriza por ser altamente productivo en carne de bovino, sin embargo, todavía existe poca información sobre la cantidad de carbono que pueden almacenar los pastizales ganaderos tropicales. Para poder conocer la función de estos agrosistemas como mitigadores del carbono atmosférico, es necesario conocer su capacidad de almacenamiento de carbono. Por lo anterior, debido a la escasez y heterogeneidad de estimaciones sobre el almacenamiento de biomasa y carbono en los pastizales tropicales, particularmente de las raíces, el presente trabajo tuvo como fin incrementar la información existente sobre carbono almacenado en la fracción radicular 
mediante la extracción directa de la biomasa, 1) total y aérea, y 2) radicular en su distribución vertical, en un área ganadera del estado de Tabasco, con la finalidad de contribuir también al mapa geográfico de México de reservas de carbono de los agrosistemas antrópicos, y con esto al mapa mundial de los almacenes de carbono radicular, dada su importancia actual.

\section{MATERIALES Y MÉTODOS}

Área de estudio: El trabajo se realizó entre agosto y octubre 2016 en dos agrosistemas de pastizal con más de 40 años de uso, a un costado del relicto de selva mediana subperennifolia del Parque Yumka, en el Ejido Dos Montes a $17 \mathrm{~km}$ al este de Villahermosa, Centro, Tabasco $\left(17^{\circ} 59^{\prime} 10^{\prime \prime}-18^{\circ} 00^{\prime} 50^{\prime \prime} \mathrm{N} \&\right.$ 92॰47'41"'-9249'20”' O). Los pastos dominantes fueron: Paspalum notatum Flugge y Urochloa decumbens Stapf (sinón. Brachiara decumbens Stapf). Paspalum notatum ("remolino", "estrella", "gramas") es una especie nativa de México y América, y U. decumbens es una especie introducida de África. La primera se caracteriza por presentar un crecimiento vegetativo en estolones (tallos rastreros, pocos individuos con muchas raíces en común), y $U$. decumbens un crecimiento vegetativo en macollos con muchos individuos separados con raíz individual. Ambos presentan metabolismo del carbono $\mathrm{C}_{4}$ (Klink \& Joly, 1989).

En cada pastizal, con la ayuda de fotomapas (INEGI, 1986) y visitas de campo, se ubicaron tres parcelas de $1600 \mathrm{~m}^{2}(40 \times 40$ m) cada una, considerando que fueran lo más alejadas y similares entre sí, para que pudieran representar lo más posible repeticiones estadísticas independientes. Las parcelas presentaron relieve y tipo de suelo similar (Fluvisol y Luvisol, Palma-López \& Triano, 2002), y se ubicaron entre sí a una distancia mínima de 300 $\mathrm{m}$ y máxima de $1500 \mathrm{~m}$. Con el apoyo de los dueños de las tierras se realizó la exclusión del ganado de estas áreas de pastoreo durante tres meses para permitir que el pasto se recuperara y creciera a su máxima altura, permitiendo su floración y fructificación. En la región es una práctica común la quema de pasto viejo para su renovación, sin embargo, no es una constante siempre y en esta ocasión no hubo registro alguno de haberse presentado.

Diseño experimental: El diseño experimental de todo el estudio se basó en el uso de tres parcelas en cada tipo de pastizal. Dentro de cada parcela, las unidades de muestreo para el estudio de la vegetación, y estimación de la biomasa y carbono aéreo fueron diez subcua-

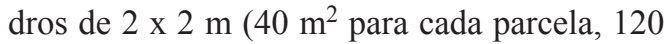
$\mathrm{m}^{2}$ por pastizal), mientras que para la estimación de la biomasa y carbono radicular fueron tres trincheras de forma rectangular de $1.50 \mathrm{~m}$ x $0.50 \mathrm{~m} \times 1.0 \mathrm{~m}$ de profundidad, dando un total de 9 trincheras para cada tipo de pastizal $\left(6.75 \mathrm{~m}^{2}\right)$. Todas las unidades de muestreo fueron distribuidos al azar dentro de cada parcela, estableciendo un perímetro con una cuerda marcada cada $\mathrm{m}$ y seleccionando coordenadas adentro con una tabla de números al azar. Para cada pastizal, el valor por hectárea de biomasa y carbono almacenado, se obtuvo extrapolando a una hectárea el valor correspondiente en 120 $\mathrm{m}^{2}$ para la parte aérea, y $6.75 \mathrm{~m}^{2}$ para la parte radicular. La suma de la biomasa aérea más la biomasa radicular representó la biomasa total, y la suma del carbono aéreo más el carbono radicular representó el carbono total. Para la composición florística de los pastizales no fue necesario realizar análisis estadístico, a diferencia de la comparación de su biomasa y almacenes de carbono.

\section{Descripción de la composición florísti-} ca y estructura de los sistemas de pastizal: Topográficamente cada parcela fue uniforme, aunque hubo cierta variación de su composición florística entre ellas. Para determinar la dominancia de las especies, se estimó el porcentaje de cobertura que ocupaban en cada subcuadro de $4 \mathrm{~m}^{2}$ de acuerdo a la escala de dominancia de Braun-Blanquet (1979), cuya escala cualitativa va de cobertura escasa hasta $100 \%$ de cobertura del área o cuadro, con valores relativos de 1 y 10 , respectivamente. Se realizaron colectas de las morfo-especies 
presentes en las unidades de muestreo para su posterior determinación en el herbario de la Universidad Juárez Autónoma de Tabasco.

Una vez identificadas las especies, para comparar los sistemas de pastizal se usó el coeficiente de similitud florística de Sorensen (Franco et al., 1995) que se expresa como:

$$
C s=\frac{2 c}{a+b} \times 100
$$

Donde:

$\mathrm{a}=$ Número de especies en el pastizal de

B. decumbens.

$\mathrm{b}=$ Número de especies en el pastizal de

P. notatum.

$\mathrm{c}=$ Número de especies que se presentan en ambos pastizales.

\section{Muestreo de la biomasa aérea y radicu-}

lar: Después de 120 días sin pastoreo y hasta la presencia de floración, se evaluó la cantidad máxima acumulada de biomasa aérea, biomasa radicular y carbono almacenado de cada pastizal. La biomasa aérea en cada uno de los subcuadros se obtuvo mediante un corte con machete al ras de suelo. La vegetación cortada se colocó en bolsas de papel, se registró el peso fresco y se trasportó al laboratorio para su secado en horno a una temperatura de $70{ }^{\circ} \mathrm{C}$ durante $72 \mathrm{~h}$.

La biomasa subterránea también se estimó en forma directa. Las trincheras fueron excavadas en intervalos de $0.10 \mathrm{~m}$ de profundidad donde se tomaron diez submuestras de $0.15 \mathrm{~m}$ x $0.15 \mathrm{~m}$ x $0.10 \mathrm{~m}$ con una pala recta hasta llegar a un metro de profundidad (Hughes et al., 1999). Posteriormente el contenido de biomasa de la submuestra se extrapoló al volumen de suelo por horizonte $(1.50 \mathrm{~m} \times 0.50 \mathrm{~m} \times 0.10$ $\mathrm{m})$. Cada submuestra por cada horizonte del suelo se transportó en bolsas de plástico al laboratorio para la separación de las raíces con la ayuda de un tamiz de malla fina de $0.2 \mathrm{~mm}$ para retener las raíces finas. Todas las raíces se lavaron con agua corriente para desprenderlas del suelo adherido. Posteriormente se guardaron en bolsas de papel para determinar su peso fresco y posteriormente secarlas en un horno a $70{ }^{\circ} \mathrm{C}$ durante 72 horas. Finalmente se empleó el factor de corrección por pérdida de raíz de 1.25 a los datos finales (MacDiken, 1997).

\section{Estimación del almacén de carbono}

Para determinar el contenido de carbono orgánico en la biomasa aérea y radicular de los dos sistemas de pastizales se determinó la materia orgánica (MO) de la biomasa seca con el método por ignición a una temperatura promedio de $550{ }^{\circ} \mathrm{C}$ durante 3 horas (Nelson et al., 1996). La determinación de la MO en la biomasa aérea se realizó a partir de las cinco especies más dominantes de acuerdo a la escala de dominancia para cada sistema de pastizal, quedando constituido el número de muestras para cada parcela, en 15. Para la determinación de la MO de la biomasa radicular se tomaron muestras de las tres trincheras de cada parcela de cada uno de los horizontes de los dos sistemas de pastizal. Las muestras fueron de $3 \mathrm{~g}$ de material seco pulverizado con un molino para muestras vegetales (Marca: Janke \& Kunkel, modelo: A10). Posteriormente, para los cálculos de contenido de MO de cada muestra, se utilizó la siguiente formula (Jiménez \& García, 1992):

$$
\% \mathrm{MO}=\frac{\mathrm{PMS}-\mathrm{PMI}}{\mathrm{PMS}} \times 100
$$

Donde:

$\% \mathrm{MO}=$ Porcentaje de materia orgánica de la muestra incinerada.

PMS $=$ Peso de muestra seca.

PMI $=$ Peso de muestra incinerada.

Con el porcentaje de MO se obtuvo el contenido de carbono orgánico total (COT), con el factor de transformación de Van Bemmelen modificado por Jiménez y García (1992) que parte del supuesto de que la MO contiene $58 \%$ de carbono. El contenido de COT se expresa como:

$$
\% \operatorname{COT}=\frac{\% \mathrm{MO}}{1.84}
$$


Donde:

$\%$ COT $=$ Porcentaje de carbono orgánico de la muestra incinerada.

$\% \mathrm{MO}=$ Porcentaje de materia orgánica de la muestra incinerada.

$1.84=$ Factor Van Bemmelen corregido.

Análisis estadístico: Para la comparación de la biomasa y carbono acumulado en la parte aérea y radicular de cada especie de gramínea (variables respuesta), la unidad experimental la constituyó la parcela conformando así tres réplicas independientes para cada pastizal. Los valores de cada parcela los constituyeron el promedio de diez muestras de biomasa y carbono aéreo, y tres muestras de biomasa y carbono radicular. Se usó un ANOVA de dos factores con la intensión de comparar los dos sistemas de pastizal (primer factor, 2 niveles), y la parte morfológica de la gramínea donde se acumula más biomasa y carbono (segundo factor; parte aérea y radicular). Para el análisis de la biomasa y carbono radicular en relación a la profundidad del suelo, se usó un ANOVA de dos factores para comparar el tipo de gramínea del pastizal (primer factor, 2 niveles) y diez profundidades de suelo de $10 \mathrm{~cm}$ cada una (segundo factor, 10 niveles) (Steel \& Torrie, 1980). Adicionalmente se usó la prueba de $t$-student para la comparación de la biomasa y carbono acumulado entre los dos sistemas de pastizal. Para la comparación de medias del análisis se seleccionó a la prueba de Tukey. En todos los casos se usó un valor mínimo de prueba de hipótesis de $\mathrm{p} \leq 0.05$. Se usó STATGRAPHICS® Centurion XV.

\section{RESULTADOS}

Diversidad, composición y estructura de la vegetación en los sistemas de pastizal: En el pastizal de $U$. decumbens se encontró un total de 38 especies herbáceas correspondientes a 16 familias. En el pastizal de P. notatum se encontró un total de 40 especies herbáceas correspondientes a 19 familias (Apéndice digital 1, Apéndice digital 2).
El índice de similitud de Sorensen entre los dos tipos de pastizales fue bajo (28.2 \%). En el pastizal de $U$. decumbens esta especie tuvo una dominancia del 50 al $75 \%$ de cobertura, seguida de Axonopus compressus (Sw.) P. Beauv., Cynodon dactylon (L.) Pers. y Eleusine indica (L.) Gaertn. con una cobertura del 25 al $33 \%$. En el pastizal de P. notatum se encontró una dominancia de la especie del $75 \%$ de cobertura, seguida de Panicum maximum Jacq. con una cobertura del 33 al 55 \%; Andropogon bicornis Forssk. y Euphorbia hirta L. con una cobertura del 25 al $33 \%$; Phaseolus lathyroides L. con una cobertura cerca del $20 \%$. A pesar de estar separados los pastizales por una distancia mínima de $300 \mathrm{~m}$ de distancia, la zona mostró una alta diversidad de especies herbáceas con muy poca similitud florística.

Biomasa y carbono aéreo, radicular y total: Los resultados se presentan en promedio de $\mathrm{g} \mathrm{m}^{-2}( \pm \mathrm{DS})$ que equivale a $0.01 \mathrm{Mg}$ $\mathrm{ha}^{-1}$. La biomasa aérea de ambos pastizales no presentó diferencias significativas (550.19 \pm 133.46 DS, P. notatum; $367.21 \pm 194.31 \mathrm{DS}$, $U$. decumbens; Student, $\mathrm{t}=-2.34, \mathrm{P}=0.37)$, al igual que el carbono aéreo $(255.13 \pm 61.80 \mathrm{DS}$, $U$. decumbens; $194.31 \pm 58.20 \mathrm{DS}, P$. notatum; Student, $\mathrm{t}=-2.89, \mathrm{P}=0.37$ ). Por el contrario, la biomasa radicular total contenida a $1 \mathrm{~m}$ de profundidad del pastizal de $P$. notatum fue mayor (6 097.3 \pm 819.6 DS, Student, $\mathrm{t}=-7.17$, $\mathrm{P}=0.002)$ que la del pastizal de $U$. decumbens (1 $640.51 \pm 677.5$ DS). El carbono radicular contenido en el mismo perfil de $1 \mathrm{~m}$ de profundidad de ambos pastizales también fue mayor para P. notatum (3 $897.3 \pm 957.24 \mathrm{DS}$ ) que $U$. decumbens (1 140.2 \pm 746.59 DS, Student, $\mathrm{t}=$ $-6.19, \mathrm{P}=0.0002$ ) (Fig. 1).

La biomasa radicular total fue mayor que la biomasa aérea total (3 $745 \pm 519.6$ DS vs. $480 \pm 107.52 \mathrm{DS} \mathrm{g} \mathrm{m}{ }^{-2}$, Anova, $\mathrm{F}=111.2, \mathrm{P}=$ 0.0000 ), y el carbono radicular total fue mayor que el carbono aéreo total $\left(\begin{array}{ll}1 & 201 \pm 397.24\end{array}\right.$ DS vs. $239 \pm 46.59 \mathrm{DS}$, Anova, $\mathrm{F}=109.5, \mathrm{P}$ $=0.0000)$. Finalmente, la biomasa total de $P$. notatum fue mayor que la de $U$. decumbens (6 $621 \pm 619.6$ DS vs. $2021 \pm 507.52$ DS g m${ }^{-2}$, 
Anova, $\mathrm{F}=55.2, \mathrm{P}=0.001)$, y el carbono total de $P$. notatum fue mayor que el de $U$. decumbens $(4201 \pm 597.24$ DS vs. $1439 \pm 746.59$ $\mathrm{DS}$, Anova, $\mathrm{F}=52.4, \mathrm{P}=0.0001)$.

Distribución vertical de biomasa y $\mathrm{C}$ radicular: La distribución de la biomasa radicular disminuyó conforme aumentó la profundidad del suelo en ambos pastizales. Para ambas especies, la biomasa y el carbono fue mayor en el horizonte de $0-10 \mathrm{~cm}$ que en el horizonte de $10-40 \mathrm{~cm}$ y de $40-100 \mathrm{~cm}$. Sus respectivos valores promedio para el carbono fueron 72, 7.9 y $0.9 \%$ (Anova, $\mathrm{F}=17.8, \mathrm{P}=$ 0.008 , Fig. 2). No se midió la longitud de las raíces, sin embargo, se observó que las raíces más largas pertenecían a los pastos y llegaban a los $90 \mathrm{~cm}$ de profundidad. El $72 \%$ se ubicó en los primeros $0.1 \mathrm{~m}$ de profundidad, el 96 $\%$ de 0 a $0.5 \mathrm{~m}$, y el $4 \%$ de 0.5 a $1 \mathrm{~m}$ de profundidad (Fig. 2). La biomasa radicular de $U$. decumbens a $0.1 \mathrm{~m}$ de profundidad fue de 215 $\mathrm{g} \mathrm{m}^{-2}$ y de $150 \mathrm{~g} \mathrm{~m}^{-2}$ a $0.8 \mathrm{~m}$, mientras que la de $P$. notatum fue de $2100 \mathrm{~g} \mathrm{~m}^{-2} \mathrm{y}-1400 \mathrm{~g} \mathrm{~m}^{2}$, respectivamente. El carbono para $U$. decumbens

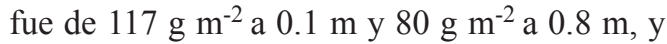
para $P$. notatum de 1130 y $400 \mathrm{~g} \mathrm{~m}^{-2}$ a 0.1 y 0.8 $\mathrm{m}$ respectivamente. El $\mathrm{C}$ radicular almacenado por $P$. notatum a $0.50 \mathrm{~m}$ de profundidad fue mayor que el de $U$. decumbens (1.86 $\mathrm{Mg} \mathrm{C} \mathrm{ha}^{-1}$ vs. $\left.1.2 \mathrm{C} \mathrm{ha}^{-1}\right)$.

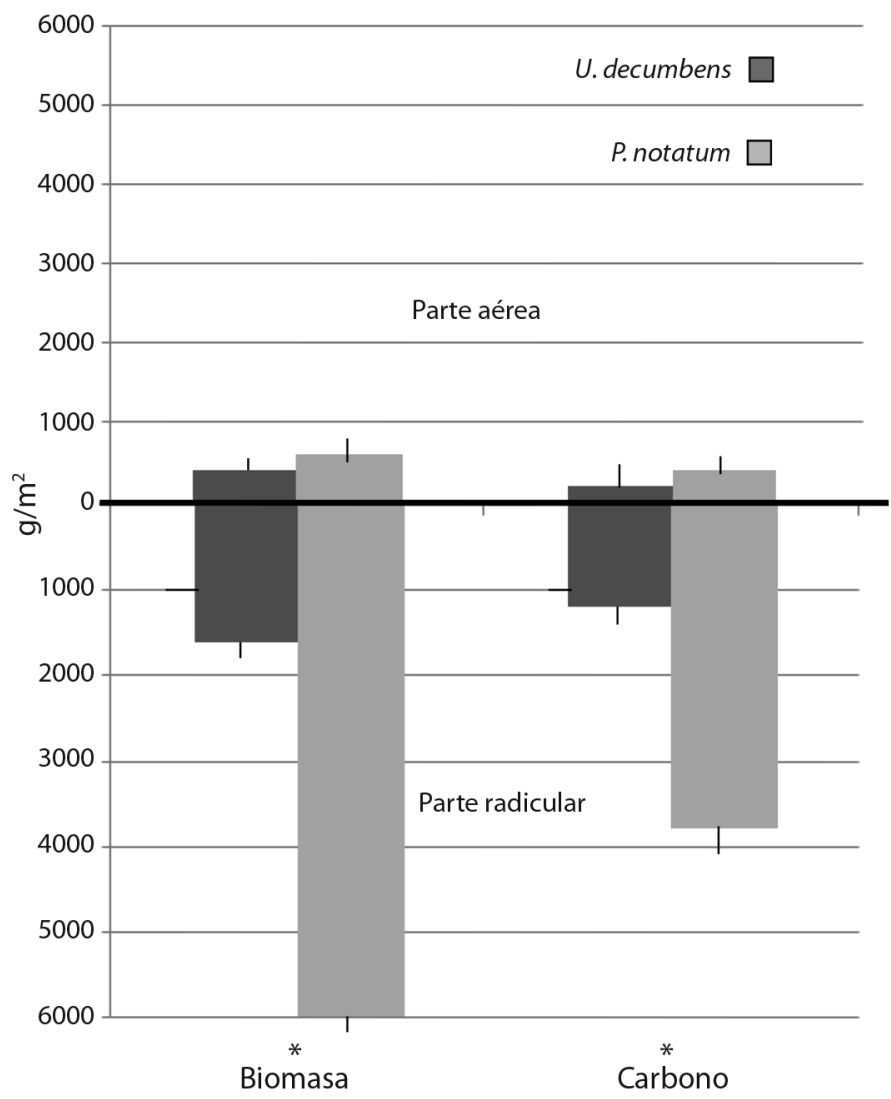

Fig. 1. Almacenes de biomasa y carbono de $U$. decumbens y $P$. notatum en la planicie de Tabasco, México. * $=$ diferencia significativa (Student, $\mathrm{P}<0.05$ ) entre las dos especies en la parte radicular.

Fig. 1. Biomass and carbon stocks for $U$. decumbens and $P$. notatum in the Tabasco plain, México. $*=$ significant difference (Student, $\mathrm{P}<0.05$ ) between the two species in the root part. 


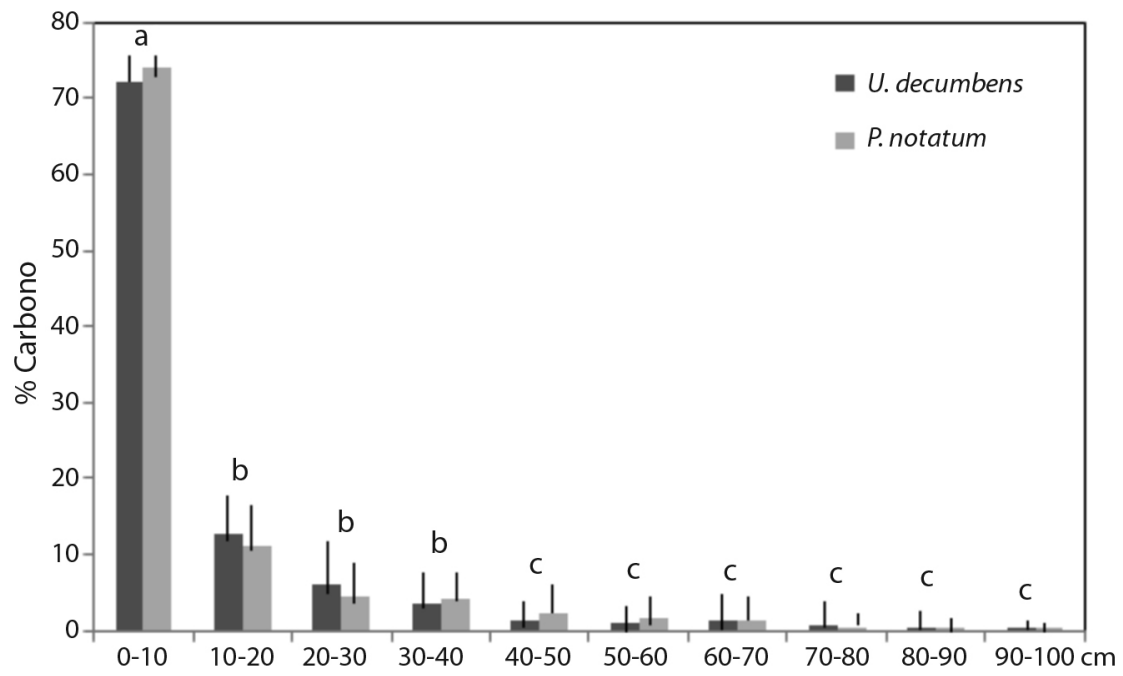

Fig. 2. Variación del carbono radicular con la profundidad del suelo para cada tipo de pastizal. Diferentes letras significan valores diferentes de carbono entre la profundidad de suelo (Anova, $\mathrm{F}=17.8, \mathrm{P}=0.008$ ).

Fig. 2. Root carbon variation with soil depth for each type of grassland. Different letters mean different values of carbon between the soil depth (Anova, $\mathrm{F}=17.8, \mathrm{P}=0.008$ ).

\section{DISCUSIÓN}

La composición florística de las parcelas dominadas por los dos tipos de pastos presentó diferencias muy probablemente debido a la historia de manejo de los sitios, tales como la edad, las actividades agropecuarias realizadas y su duración, ya que ambas parcelas experimentaron en forma distinta cultivo maíz, legumbres y pasto para forraje. Debido a la edad de las parcelas, lo anterior no se pudo determinar con exactitud.

Los valores encontrados en este estudio, están dentro del rango de valores para estudios similares reportados por otros autores en América tropical básicamente. La biomasa total de $P$. notatum coincidió con la registrada por Hernández, Simón y Duquesne (2001) para un solo corte en épocas de lluvias. Los almacenes de $\mathrm{C}$ en la parte aérea de los pastizales de $P$. notatum ( $\left.2.55 \mathrm{MgC} \mathrm{ha}^{-1}\right)$ y $U$. decumbens $\left(1.94 \mathrm{MgC} \mathrm{ha}^{-1}\right)$ fueron similares a las pasturas de $B$. brizanta $\left(2.0 \pm 0.1 \mathrm{MgC} \mathrm{ha}^{-1}\right)$ en Costa Rica (Ávila et al., 2001), de Huatusco, Veracruz, México (1.78 $\mathrm{MgC} \mathrm{ha}^{-1}$, Torres-Rivera,
Espinoza-Domínguez, Reddiar-Krishnamurth, \& Vázquez-Alarcón, 2011), el Parque Ecológico Jaguaroundi en Coatzacoalcos, México (3.0 $\mathrm{MgC} \mathrm{ha}{ }^{-1}$, Martínez-Bravo \& Masera, 2008), aunque menores que en el Amazonas del Perú (4.25 $\mathrm{MgC} \mathrm{ha}^{-1}$ ) (Concha, Krishnamurthy, \& Alegre, 2002). De Jong, Ochoa-Gaona, Castillo-Santiago, Ramírez-Marcial y Cairns (2000) estimaron hasta $23.2 \mathrm{MgC} \mathrm{ha}^{-1}$ para los pastizales de los Altos de Chiapas, México, aunque el valor se debe tomar con mucha reserva debido a que se estimó con fórmulas alométricas.

La biomasa y $\mathrm{C}$ radicular estimados para $P$. notatum $\left(60.9,38.0 \mathrm{Mg} \mathrm{ha}^{-1}\right)$ y $U$. decumbens $\left(16.4,12.2 \mathrm{Mg} \mathrm{ha}^{-1}\right)$ a una profundidad de $1 \mathrm{~m}$ resultó ser mayor a la registrada en pastizales de los Tuxtlas, Veracruz (3.1-5.45, 0.97-3.25 $\mathrm{Mg} \mathrm{ha}^{-1}$; Hughes et al., 1999), aunque su distribución en relación a la profundidad del suelo fue similar, siendo mayor en los horizontes superficiales $(90 \%$ en los primeros $40 \mathrm{~cm}$ ). Probablemente, lo anterior se deba a la diferencia del suelo ya que en los Tuxtlas, Veracruz, los suelos son Andosoles altamente fértiles, siendo tal vez por esta razón innecesario el desarrollo 
de gran biomasa subterránea. Similarmente, Ramírez, Ramírez, \& Suárez (2009) encontraron que el $80 \%$ de las raíces de $U$. decumbens se encuentran en los primeros $10 \mathrm{~cm}$ de profundidad y el $20 \%$ en los siguientes $90 \mathrm{~cm}$. Se ha encontrado relación entre el tamaño de la raíz y la textura del suelo. Callejas-Rodríguez, Rojo-Torres, Benavidez-Zabala y Kania-Kuhl (2012) encontraron que en suelos con textura gruesa las raíces de la vid fueron superficiales, mientras en suelos de textura media, fueron más profundas. Gaitán, Penón y Costa (2005) encontraron en Eucalyptus globulus spp. maidenii una relación negativa entre tamaño de la raíz y contenido de arcilla y densidad aparente del suelo. Dada la alta densidad de biomasa radicular existente en los pastizales, se puede explicar las grandes cantidades de carbono orgánico en el suelo en diferentes regiones del mundo como Costa Rica y Cuba para Panicum maximum (60.4, $43.7 \mathrm{Mg} \mathrm{ha}^{-1}$; Lok, Fraga, Noda, \& García 2013), Perú $93.01 \mathrm{Mg} \mathrm{ha}^{-1}$ (Concha et al., 2002), y China $48.9 \mathrm{Mg} \mathrm{ha}^{-1}$ (Kong, Shi, \& Chu 2014).

Mediante el uso de fórmulas alométricas diversos autores han estimado almacenes de $\mathrm{C}$ radicular a $1 \mathrm{~m}$ de profundidad. Concha et al. (2002) en dos sitios de Perú, estimaron 0.68 $\mathrm{MgC} \mathrm{ha}{ }^{-1}$ en pastura de $U$. decumbens degradada de 1 año, $0.96 \mathrm{Mg} \mathrm{C}^{-1}$ en pastura de 15 años, y $1.50 \mathrm{MgC} \mathrm{ha}^{-1}$ en pastura de 30 años. De Jong et al. (2000) estimaron almacenes desde 4.1 a $27.0 \mathrm{MgC} \mathrm{ha}^{-1}$ en los Altos de Chiapas, México, y Delaney \& Roshetko (1999) de $5.1 \mathrm{MgC} \mathrm{ha}^{-1}$ para pastizales de Filipinas. Los métodos para medición de biomasa radicular de los ecosistemas no están bien establecidos, y debido a su dificultad como a la alteración del suelo, la mayoría de los trabajos no la estiman a través de la extracción directa. Delaney, Brown, Lugo, Torre y Quintero (1997) registraron un espectro de variación considerable de $22 \%$ calculando los valores por medio de un cociente raíz-tallo promedio y no por cuantificación directa. Cairns, Brown, Helmer y Baumgardner (1997) a partir de datos de biomasa aérea y con ayuda de ecuaciones de regresión estimaron variaciones muy amplias de biomasa radicular para las zonas tropicales (1-128 $\left.\mathrm{Mg} \mathrm{ha}^{-1}\right)$. En este trabajo se realizó una extracción directa ya que arroja datos más precisos que los métodos indirectos. Estos almacenes de carbono fijo pueden tener secuestrado $\mathrm{CO}_{2}$ atmosférico en los suelos del ecosistema a través de la deposición y descomposición de la hojarasca, raíces, exudados de las raíces, y otros materiales, lo cual se correlaciona positivamente con la precipitación (Eaton, McGoff, Byrne, Leahy, \& Kiely 2008; Nian-Peng, Ruo-Meng, Zhang, \& Quan-Sheng 2014). Sin embargo, es importante adicionar la respiración del suelo como una fuente negativa importante de emisiones de carbono (Kong, 2014).

Claramente, todos los pastizales retienen menos carbono en su biomasa total que la vegetación arbórea que les precedió, que en la Amazonia brasileña llega a ser de $132-554 \mathrm{Mg}$ ha $^{-1}$ (Houghton et al., 2000). La conversión de vegetación arbolada a pastizales representa una pérdida considerable de carbono almacenado (Jaramillo et al., 2003; de Koning et al. 2003). En esta región, el carbono total promedio almacenado en la biomasa del pastizal fue alrededor del $42 \%$ (28.25 $\left.\mathrm{Mg} \mathrm{ha}^{-1}\right)$ del almacenado en la biomasa aérea de la vegetación arbolada de la misma zona (67.5 MgC hä ${ }^{-1}$, Martínez-Sánchez \& Cámara, 2012). Sin embargo, su importancia radica en la mayor extensión que actualmente presentan los pastizales en comparación con la vegetación arbórea. Además, hay que tener en cuenta que existe una ventaja relativa en el reemplazo de vegetación arbolada de metabolismo $\mathrm{C}_{3}$ por vegetación herbácea de metabolismo $\mathrm{C}_{4}$ como la mayoría de las especies de pastos tropicales (Klink \& Joly, 1989), representando una ganancia relativa de carbono por área foliar o fotosintética (Veldkamp, 2003). Los pastos $\mathrm{C}_{4}$ presentan mayor contenido de azúcares, relación azúcares/ $\mathrm{N}$ y menor contenido de nitrógeno que los pastos $\mathrm{C}_{3}$, por lo que dan mayor capacidad de integrar el $\mathrm{CO}_{2}$ en la materia orgánica que los pastos $\mathrm{C}_{3}$ (Cajarville et al., 2007).

El pastizal de $P$. notatum presentó mayor biomasa total que el pastizal de $U$. decumbens, siendo la biomasa radicular el componente 
principal para el almacenamiento de C. El patrón general de distribución de biomasa de ambas especies se puede describir como una biomasa aérea similar pero una mayor biomasa total de $P$. notatum debido a una mayor biomasa radicular. Considerando que el tipo de suelo principalmente en lo que se refiere a sus propiedades físicas puede afectar a la biomasa radicular, y que los dos sistemas silvopastoriles presentaron el mismo tipo de suelo, se puede pensar que las diferencias en biomasa radicular entre las dos especies pueden estar dadas por la diferencia morfológica en su sistema radicular, ya que $U$. decumbens presenta un crecimiento en macollos (separación entre individuos) mientras que $P$. notatum presenta crecimiento en estolón, lo cual le permite cubrir más área con un mismo individuo. Así también, $U$. decumbens es un pasto introducido mientras que $P$. notatum es nativo, lo que puede ser otra razón por la que $P$. notatum tenga una mayor productividad al estar mejor adaptado sobre todo a climas secos. Se ha documentado la menor productividad de especies introducidas en comparación con las especies nativas (ver Meléndez, 2003). Urochloa decumbens en Tabasco, México, está por debajo de su capacidad para producir biomasa seca, la cual según Meléndez (2003) es de $25 \mathrm{Mg} \mathrm{ha}^{-1} \mathrm{año}^{-1}$ para un clima tropical.

Como se comentó en la sección de metodología, es común el uso del fuego para la renovación del pasto en los pastizales ganaderos. Al respecto, Giorgis, Cingolani y Cabido (2013) encontraron que el factor más importante en la disminución de la composición florística y biomasa de la vegetación de un pastizal, fue el tiempo transcurrido desde la última quema, ya que la resiliencia del agrosistema prácticamente anula el efecto negativo de quemas antiguas. En este sentido, dado que no se encontró evidencia de alguna quema reciente en las parcelas estudiadas a partir de sus propietarios, se puede decir que los resultados aquí encontrados no están influenciados por el efecto de los incendios.

Finalmente, la mayor productividad de $P$. notatum se puede decir que tal vez no representa una ventaja para el sector ganadero al no proveer mayor biomasa forrajera, sino más bien una posible adaptación a climas secos. El estado de Tabasco posee un clima tropical húmedo, donde ocurre escasez de agua solo en la primavera, con valores de precipitación mayores que en la región ganadera del norte de México, donde también se ha adaptado este pasto. Quizás un pasto adaptado a un clima húmedo y con mayor productividad de biomasa aérea sea una mejor opción para el estado de Tabasco, sobre todo considerando que el manejo y la carga animal (consumo de biomasa aérea) afectan poco o no afectan la biomasa y el carbono radicular, particularmente en $U$. decumbens (Saraiva et al., 2014). Desde el punto de vista de producción ganadera, tal vez no es muy recomendable el uso de $P$. notatum debido a su menor producción foliar que radicular, aunque con fines de conservación del carbono si sería recomendable.

Como resultado de este trabajo, se puede concluir que el almacenamiento de $\mathrm{C}$ en los pastizales de $P$. notatum es de $74.5 \%$ en la parte radicular y $25.5 \%$ en la parte aérea, mientras que en los pastizales de $U$. decumbens es de 56.5 y $43.5 \%$, respectivamente. Esta mayor productividad de $P$. notatum puede estar asociada a su forma de crecimiento vegetativo en estolones y a su adaptación como especie nativa.

Declaración de ética: los autores declaran que todos están de acuerdo con esta publicación y que han hecho aportes que justifican su autoría; que no hay conflicto de interés de ningún tipo; y que han cumplido con todos los requisitos y procedimientos éticos y legales pertinentes. Todas las fuentes de financiamiento se detallan plena y claramente en la sección de agradecimientos. El respectivo documento legal firmado se encuentra en los archivos de la revista.

\section{RESUMEN}

Introducción: Debido a su extensión actual, la biomasa radicular de los pastizales tropicales es de vital importancia 
para el almacenamiento del carbono terrestre, pero su estimación ha sido principalmente indirecta y con un rango muy amplio de valores. Objetivo: Documentar a nivel nacional y mundial, y comparar, las existencias de carbono radicular extraídas en forma directa, de dos pastizales de origen y tipo de crecimiento distinto. Método: Se midió la biomasa y carbono total de dos pastizales con más de 40 años de uso, uno nativo de América y de crecimiento estolonífero (Paspalum notatum) y otro introducido de África y de crecimiento en macollos (Urochloa decumbens). El estudio se realizó entre agosto y octubre 2016. Se seleccionaron 3 parcelas de $1600 \mathrm{~m}^{2}$ por pastizal, con 10 subcuadros $\left(4 \mathrm{~m}^{2} \mathrm{c} / \mathrm{u}\right)$ en cada parcela, para determinar composición florística, biomasa y $\mathrm{C}$ aéreo. La biomasa y $\mathrm{C}$ radicular se estimaron mediante extracción directa en tres trincheras $(1.50 \mathrm{~m} \times 0.50 \mathrm{~m} \mathrm{x} 1.0 \mathrm{~m})$ por parcela. El contenido de carbono orgánico se determinó con el método por ignición a una temperatura promedio de $550{ }^{\circ} \mathrm{C}$ durante 3 horas. Para el análisis estadístico se usó un ANOVA de dos factores, en el que un factor fueron dos tratamientos (tipo de pastizal) y el otro factor fue la parte morfológica del pasto (aéreo y radicular). Resultados: La composición florística de los dos tipos de pastizal fue diferente debido a su historia de manejo. En promedio se estimó $28.25 \mathrm{MgC} \mathrm{ha}^{-1}$ total para la localidad. El pastizal nativo y de crecimiento estolonífero $P$. notatum produce casi tres veces más biomasa (42.5 $\left.\mathrm{MgC} \mathrm{ha}^{-1}\right)$ que el introducido y de crecimiento en macollos $U$. decumbens $\left(14 \mathrm{MgC} \mathrm{ha}^{-1}\right)$ debido al almacén radicular (38.5 vs. $11.46 \mathrm{MgC} \mathrm{ha}^{-1}$ ). El $74.5 \%$ del carbono en $P$. notatum se localizó en la parte radicular y el $25.5 \%$ en la parte aérea, mientras que en $U$. decumbens fueron 56.5 y $43.5 \%$, respectivamente. Conclusión: $P$. notatum acumuló más biomasa y carbono total y radicular que $U$. decumbens. La mejor adaptación del pasto nativo, así como la morfología de su sistema radicular, pueden explicar esta diferencia. El $96 \%$ de la biomasa y del C radicular se encuentra en los primeros $0.50 \mathrm{~m}$ de profundidad. Debido a la contribución de su porción radicular, los pastizales tropicales pueden constituir importantes reservorios de carbono terrestre, considerando su extensión hoy en día.

Palabras clave: Brachiaria decumbens, biomasa radicular, pasto $\mathrm{C} 4$, perfil de suelo, pastizal.

\section{REFERENCIAS}

Alegre, J., Arevalo, L., \& Ricse, A. (2000). Reservas de carbono según el uso de la tierra en dos sitios de la Amazonia Peruana. En Agroforestería para la producción animal en América Latina (p. 9). II Memorias de la segunda conferencia electrónica (Agosto 2000-Marzo 2001).

Ávila, G., Jiménez, F., Beer, J., Gómez, M., \& Ibrahim, M. (2001). Almacenamiento, fijación de carbono y valoración de servicios ambientales en sistemas agroforestales en Costa Rica. Agroforestería en las Américas, 8(30), 32-35.
Barbaran, J., Rise, A., \& Alegre, J. (2001). Cuantificación de biomasa y carbono en sistemas de uso del suelo de Campo Verde, Ucayali, Perú. En Simposio Internacional Medición y Monitoreo de la captura de carbono en ecosistemas forestales. Valdivia, Chile.

Braun-Blanquet, J. (1979). Fitosociología: Bases para el estudio de las comunidades vegetales. Madrid, España: Blume.

Cairns, M.A., Brown, S., Helmer, E.H., \& Baumgardner, G.A. (1997). Root biomass allocation in the world's upland forests. Oecologia, 111, 1-11. DOI: 10.1007/ s004420050201

Cajarville, C., Britos, A., Caramelli, A., Antúnez, M., Zanoniani, R., Boggiano, P., \& Repetto, J.L. (2007). El horario de corte y el tipo de metabolismo fotosintético afectan la relación azúcares/nitrógeno de las pasturas. Sitio Argentino de Producción Animal (1-5). Recuperado de http://www.produccion-animal. com.ar/produccion_y_manejo_pasturas/pastoreo $\% 20$ sistemas/89-Cajarville.pdf

Callejas-Rodríguez, R., Rojo-Torres, E., Benavidez-Zabala, C., \& Kania-Kuhl, E. (2012). Crecimiento y distribución de raíces y su relación con el potencial productivo de parrales de Vides de Mesa. Agrociencia, 46(1), 23-35.

Carrillo, A.F., Acosta, M.M., Flores, A.E., Juárez, B.J., \& Bonilla, P.E. (2014). Estimación de biomasa y carbono en dos especies arbóreas en La Sierra Nevada, México. Revista Mexicana de Ciencias Agrícolas, 5(5), 779-793.

Castro, T.A. (2008). Estimación de secuestro de carbono en pastizales áridos (Tesis de Licenciatura). Universidad Autónoma Agraria Antonio Narro, México.

Céspedes, F.E.F., Fernández, J.A., Gobbi, J.A., \& Bernardis, A.C. (2012). Reservorio de carbono en suelo y raíces de un pastizal y una pradera bajo pastoreo. Revista Fitotecnia Mexicana, 35(1), 79-86.

Concha, D.C., Krishnamurthy, L., \& Alegre, J. (2002). Secuestro de carbono por sistemas agroforestales amazónicos. Revista Chapingo Serie Ciencias Forestales y del Ambiente, 8(2), 101-106.

D’Atri, P. (2007). Pastizales del mundo. Novedades de Biodiversidad en América Latina No 160.

De Jong, B.H., Ochoa-Gaona, S., Castillo-Santiago, M.A., Ramírez-Marcial, N., \& Cairns, M.A. (2000). Carbon flux and patterns of land-use/land-cover change in the Selva Lacandona, Mexico. AMBIO: A Journal of the Human Environment, 29(8), 504-511. DOI: 10.1579/0044-7447-29.8.504

de Koning, G.H.J., Velkamp, E., \& López Ulloa, M. (2003). Quantification of carbon sequestration in soils following pasture to forest conversion in 
northwestern Ecuador. Global Biogeochemical Cycles, 17(4), 1098.

Delaney, M., \& Roshetko, J. (1999). Field test of carbon monitoring methods for homegardens in Indonesia. In Forest Carbon Monitoring Program (Ed.), Field Tests of Carbon Monitoring Methods in Forestry Projects (pp. 45-51). Arlington, USA: Winrock International.

Delaney, M., Brown, S., Lugo, A.E., Torre, A., \& Quintero, N.B. (1997). The distribution of organic carbon in major components of forests located in five life zones of Venezuela. Journal of Tropical Ecology, 13(5), 697-708. DOI: 10.1017/S0266467400010877

Dixon, A.P., Faber-Langendoen, D., Josse, C., Morrison, J., \& Loucks, C.J. (2014). Distribution mapping of world grassland types. Journal of Biogeography, 14(11), 2003-2019. DOI: 10.1111/jbi.12381

Eaton, J.M., McGoff, N.M., Byrne, K.A., Leahy, P., \& Kiely, G. (2008). Land cover change and soil organic carbon stocks in the Republic of Ireland 1851-2000. Climatic Change, 91(3-4), 317-334. DOI: 10.1007/ s10584-008-9412-2

Fan, J., Zhong, H., Harris, W., Yu, G., Wang, S., Hu, Z., \& Yue, Y. (2008). Carbon storage in the grasslands of China based on field measurements of above-and below-ground biomass. Climatic Change, 86(3-4), 375-396. doi:10.1007/s10584-007-9316-6

Franco, L., de la Cruz, A., Cruz, G., Rocha, R., Navarrete, S., Flores, M., \& Bedia, S. (1995). Manual de ecología. D.F., México: Trillas.

Gaitán, J.J., Penón, E.A., \& Costa, M.C. (2005). Distribución de raíces finas de Eucalyptus globulus ssp. maidenii y su relación con algunas propiedades del suelo. Ciencia Forestal, 15(1), 33-41.

Giorgis, M.A., Cingolani, A.M., \& Cabido, M.R. (2013). El efecto del fuego y las características topográficas sobre la vegetación y las propiedades del suelo en la zona de transición entre bosques y pastizales de las sierras de Córdoba, Argentina. Boletín de la Sociedad Argentina de Botánica, 48(3-4), 493-513.

Giraldo, L.A., Zapata, M., \& Montoya, E. (2006). Estimación de la captura y flujo de carbono en silvopastoreo de Acacia mangium asociada con Brachiaria dyctioneura en Colombia. Pastos y Forrajes, 29(4), 421-435.

Gómez, I.A., \& Gallopin, G.C. (1991). Estimación de la productividad primaria neta de ecosistemas terrestres del mundo en relación a factores ambientales. Ecología Austral, 1, 24-40.

Hernández, I., Simón, L., \& Duquesne, P. (2001). Evaluación de las arbóreas Albizia lebbeck, Bauhinia purpurea y Leucaena leucocephala en asociación con pasto bajo condiciones de pastoreo. Pastos y Forrajes, 24(3), 241-258.
Hernández, H.M. (2008). Secuestro de carbono en pastizales: efecto del sobrepastoreo en la heterogeneidad del suelo (Tesis de Licenciatura). Universidad Autónoma Agraria Antonio Narro, México.

Houghton, R.A., Skole, D.L., Nobre, C.A., Hackaler, J.L., Lawrance, K.T., \& Chamentowski, W.H. (2000). Annual fluxes of carbon from deforestation and regrowth in the Brazilian Amazon. Nature, 403, 301304. DOI:10.1038/35002062

Houghton, R.A., Lawrence, K.T., Hackler, J.L., \& Brown, S. (2001). The spatial distribution of forest biomass in the Brazilian Amazon: a comparison of estimates. Global Change Biology, 7(7), 731-746. DOI: 10.1111/j.1365-2486.2001.00426.x

Hughes, R.F., Kauffman, J.B., \& Jaramillo, V.J. (1999). Biomass, carbon, and nutrient dynamics of secondary forests in a humid tropical region of México. Ecology, 80 (6), 1892-1907.

DOI: $10.1890 / 0012-9658(1999) 080[1892: B C A N D O] 2.0$ $\mathrm{CO} ; 2$

INEGI. Instituto Nacional de Estadística y Geografía. México en cifras. Recuperado de www.beta.inegi.org. $\mathrm{mx} /$ app/areasgeograficas

INEGI. (1986). Fotografía aérea de Villahermosa, Tabasco. Instituto Nacional de Estadística y Geografía.

Jaramillo, V.J., Ahedo-Hernández, R., \& Kauffman, B. (2003). Root biomass and carbon in a tropical evergreen forest of Mexico: changes with secondary succession and forest conversion to pasture. Journal of Tropical Ecology, 19(4), 457-464.

Jarrige, R., Grenet, E., Demarquilly, C., \& Beslé, J.M. (1995). Les constituents de 1'appareil végétatifs des plantes fourragères. In R. Jarriage, Y. Ruckebush, C. Demarquilly, M.H. Faqree, \& M. Journet (Eds.), Nutrition des ruminants domestiques. Paris, France: INRA.

Jiménez, E.I., \& García, V.P. (1992). Relationships between organic carbon and total organic matter in municipal solid wastes and city refuse composts. Bioresource Technology, 41(3), 265-272. DOI:10.1016/0960-8524(92)90012-M

Kanninen, M., \& Mery, G. (2001). Carbon sinks in different forest ecosystems in Latin America. In M. Palo, J. Uusivouri, \& G. Mery (Eds.), World forests markets and policies (pp. 68-70). The Netherlands: Kluwer Academic Publishers.

Klink, C.A., \& Joly, C.A. (1989). Identification and distribution of $\mathrm{C} 3$ and $\mathrm{C} 4$ grasses in open and shaded habitats in Sao Paulo State, Brazil. Biotropica, 21(19), 30-34.

Kong, L., Shi, Z., \& Chu, L.M. (2014). Carbon emission and sequestration of urban turfgrass systems in Hong 
Kong. Science of the Total Environment, 473, 132138. DOI:10.1016/j.scitotenv.2013.12.012

Lok, S., Fraga, S., Noda, A., \& García, M. (2013). Almacenamiento de carbono en el suelo de tres sistemas ganaderos tropicales en explotación con ganado vacuno. Revista Cubana de Ciencia Agrícola, 47(1), 75-82.

López, A., Schlonvoight, A., Ibrahim, M., Kleinn, C., \& Kanninen, M. (1999). Cuantificación del carbono almacenado en el suelo de un Sistema silvopastoril en la zona Atlántica de Costa Rica. Agroforestería de las Américas, 6, 51-53.

Martínez-Bravo, R., \& Masera, O. (2008). La captura de carbono como servicio ecosistémico del Parque Ecológico Jaguaroundi: una estrategia para la conservación y manejo de los recursos forestales. In Y. Nava, I. Rosas, \& R. Dirzo (Eds.), El Parque Ecológico Jaguaroundi (pp. 103-114). D.F., México: Secretaría del Medio Ambiente y Recursos Naturales-Instituto Nacional de Ecología.

Martínez-Sánchez, J.L., \& Cámara, L. (2012). Is there a relationship between floristic diversity and carbon stocks in tropical vegetation in Mexico? African Journal of Agricultural Research, 7(17), 2584-2591.

Meléndez, N.F. (2003). Manejo de forrajes tropicales en Tabasco. Tabasco, México: Gobierno del estado de Tabasco-Instituto Nacional de Investigaciones Forestales, Agrícolas y Pecuarias-Instituto para el Desarrollo del Sistema de Producción del Trópico Húmedo.

Montaño, N.M., Ayala, F., Bullock, S.H., Briones, O., García, F.O., García, R.S., ... Yépez, E. (2016). Almacenes y flujos de carbono en ecosistemas áridos y semiáridos de México: Síntesis y perspectivas. Terra Latinoamericana, 34, 39-59.

Nelson, D.W., Sommers, L.E., Sparks, D.L., Page, A.L., Helmke, P.A., Loeppert, R.H., \& Sumner, M.E. (1996). Total carbon, organic carbon, and organic matter. In D.L. Sparks, A.L. Page, \& R.H. Loeppert (Eds.), Methods of Soil analysis Part 3-Chemical methods (pp. 961-1010). American Madison, Wisconsin, USA: Society of Agronomy, Inc.

Ni, J. (2002). Carbon storage in grasslands of China. Journal of Arid Environments, 50(2), 205-218. DOI: http://dx.doi.org/10.1006/jare.2001.0902

Nian-Peng, H.E., Ruo-Meng, W., Zhang, Y.H., \& QuanSheng, C. (2014). Carbon and nitrogen storage in
Inner Mongolian grasslands: relationships with climate and soil texture. Pedosphere, 24(3), 391-398. DOI: 10.1016/S1002-0160(14)60025-4

Palma-López, D., \& Triano, A.S. (2002). Plan de uso sustentable de los suelos de Tabasco. Vol. II. Villahermosa, México: Colegio de Postgraduados-ISPROTAB.

Ramírez, B.L., Ramírez, H.F., \& Suárez, J.C. (2009). Carbon sequestration and root development of systems of soil use in the Colombian Amazon. Livestock Research for Rural Development, 21(6), 1-5.

Ruolo, S.C., Pérez, A.H., Carranza, F., Pons, D., Demaría, M., Andreu, E., \& Rigonatto, G., Composición botánica y biomasa de un pastizal serrano pre y postpastoreo en Potrero de Garay, Córdoba. Recuperado de https://inta.gob.ar/sites/default/files/inta composicion_botanica_y_biomasa_de_un pastizal serrano_pre_y_postpastoreo.pdf. Accedido 2019-08-23

Saraiva, F.M., Dubeux, Jr. J.C.B., Lira, M.A., Mello, A.C.L. de Santos, M.V.F., Cabral, F.A., \& Teixeira, V.I. (2014). Root development and soil carbon stocks of tropical pastures managed under different grazing intensities. Pastos y Forrajes, 2, 254-261. DOI:10.17138/TGFT(2)254-261.

SEMARNAT. (s.f.). Secretaria del Medio Ambiente y Recursos Naturales. El medio ambiente en México 2013-2014. Recuperado de https://apps1.semarnat. gob.mx:8443/dgeia/informe_resumen14/02_ecosistemas/2_1.html

Steel, R.G., \& Torrie, J.H. (1980). Bioestadística (2a . ed.). D.F., México: MacGrawHill.

Torres-Rivera, J.A., Espinoza-Domínguez, W., ReddiarKrishnamurthy, L., \& Vázquez-Alarcón, A. (2011). Secuestro de carbono en potreros arbolados, potreros sin árboles y bosque caducifolio de Huatusco, Veracruz. Tropical and Subtropical Agroecosystems, 13(3), 543-549.

Veldkamp, E. (2003). Changes in soil carbon stocks following conversion of forest to pasture in the tropics. In E.A. Holland (Ed.), Notes from Underground: Soil Processes and Global Change (p. 20). Berlin, Germany: NATO ASI Series, Springer.

Yamallel, J.I., Pérez, J.J., Alanís, E., Rodríguez, O.A.A.C., Tagle, M.A.G., \& Garza, E.J.T. (2014). Dinámica de la captura de carbono en pastizales abandonados del noreste de México. Tropical and Subtropical Agroecosystems, 17, 113-121. 